\title{
Posiciones Mandibulares de Referencia Clínica. Una Descripción Narrativa
}

\author{
Mandibular Positions of Clinical Reference. Narrative description
}

\author{
Camila Venegas ${ }^{1,2} ;$ Constanza Farfán ${ }^{1,3}$ \& Ramón Fuentes ${ }^{1,4}$
}

\begin{abstract}
VENEGAS, C.; FARFÁN, C. \& FUENTES, R. Posiciones Mandibulares de Referencia Clínica. Una descripción Narrativa. Int. J.Odontostomat., 15(2):387-396, 2021.

RESUMEN: Las posiciones mandibulares se definen como la relación existente entre la mandíbula y la maxila observada durante su función, o bien posiciones de referencia clínica que pueden ser comparables con otras posiciones o movimientos. Se realizó una descripción narrativa de las posiciones de referencia mandibular de mayor interés clínico, donde se definen algunas de sus principales características; (1) la posición de máxima intercuspidación, está definida por el determinante anatómico dentario, tiene una adecuada reproducibilidad y sencilla ejecución, (2) la posición postural mandibular, es la menos estable, y está definida por el determinante fisiológico neuromuscular, siendo el resultado de la interacción de múltiples factores relacionados, (3) la relación céntrica fisiológica, se considera la posición más estable de la mandíbula, siendo repetible e independiente del contacto dentario, (4) la posición retruida ligamentosa, es una posición extrema por lo que no se considera fisiológica, está determinada por la tensión de la banda horizontal medial del ligamento temporomandibular, (5) la posición retruida de contacto, se describe como la posición mandibular cuando se producen los primeros contactos dentarios y la articulación temporomandibular (ATM) está en una posición retruida, se divide en forzada y no forzada, (6) la posición muscular de contacto es una posición regulada a través de diferentes mecanismos que permiten su estabilidad. Estas posiciones brindan una referencia clínica por medio de la cual se pueden evaluar distintos parámetros necesarios para de realizar diagnósticos y planificación de tratamientos, para ello es importante que el profesional tenga los conocimientos adecuados sobre las características que definen cada una de ellas, tales como su estabilidad y reproducibilidad, que son fundamentales al momento de su ejecución.
\end{abstract}

PALABRAS CLAVE: Posición de máxima intercuspidación, posición postural mandibular, posición muscular de contacto, posición retruida de contacto, posición retruida ligamentosa, relación céntrica fisiológica, dimensión vertical.

\section{INTRODUCCIÓN}

Oclusión se define como la relación de contacto entre los arcos dentarios superiores e inferiores, esta deriva de un proceso de cierre de ambos maxilares, resultado de la contracción sinérgica y coordinada de diferentes músculos (Manns \& Diaz, 1988), una oclusión anatómica normal requiere relaciones óptimas entre los dientes maxilares y mandibulares, esto quiere decir que los arcos maxilares y mandibulares deben corresponder entre sí en tamaño y forma (Moorrees, 1967). Se considera que la oclusión debe ser analizada desde el punto de vista estático y diná- mico, a través de movimientos activos producidos por el sistema motor mandibular, ya que las relaciones oclusales corresponden a cualquier movimiento mandibular en los planos del espacio en que los dientes estén en contacto (Davies \& Gray, 2001; Manns \& Diaz; Mohamed \& Christensen, 1985).

El sistema locomotor mandibular está definido por las diferentes estructuras anatómicas que lo conforman, las cuales permiten los movimientos y posturas activas y pasivas de la mandíbula (Mohamed \&

\footnotetext{
${ }^{1}$ Dental School, Research Centre for Dental Sciences (CICO), Universidad de La Frontera, Temuco, Chile.

${ }^{2}$ Master program in Dentistry, Dental School, Universidad de La Frontera, Temuco, Chile.

${ }^{3}$ Universidad Adventista de Chile.

${ }^{4}$ Department of Integral Adults Dentistry, Dental School, Universidad de La Frontera, Temuco, Chile.
} 
Christensen), toda actividad funcional de este sistema es producida por la acción de los músculos, guiados por el mecanismo neuromuscular, considerándose este como el determinante fundamental motodinámico de la oclusión, mientras que los dientes, periodonto y articulaciones temporomandibulares, son elementos de carácter pasivo (Apodaca, 2004; Manns \& Diaz).

Se han establecido posiciones dentarias para guiar a una correcta oclusión (Davies et al., 2001), así como también se conocen aquellas posiciones que dependen de la articulación temporomandibular (ATM), el sistema neuromuscular y los dientes (Tripodakis et al., 1995), este conjunto se conoce como posiciones mandibulares, y se definen como la relación existente entre la mandíbula y la maxila, que se observa frecuentemente durante la función, o bien posiciones de referencia clínica, que pueden ser comparables con otras posiciones o movimientos (Manns \& Diaz), se considera que para ser adecuadas como punto de partida o referencia en el examen y tratamiento del sistema locomotor mandibular, estas deben ser funcionalmente aceptables para el paciente y clínicamente estables y reproducibles, de tal manera de servir como guía en diferentes procedimientos clínicos (Mohamed \& Christensen; Tripodakis et al.). Estas posiciones se basan en la relación tridimensional que tiene la mandíbula con el cráneo, y su fisiología se basa en la contribución de diferentes elementos del sistema estomatognático que permiten su reproducibilidad (Brill \& Tryde, 1974).

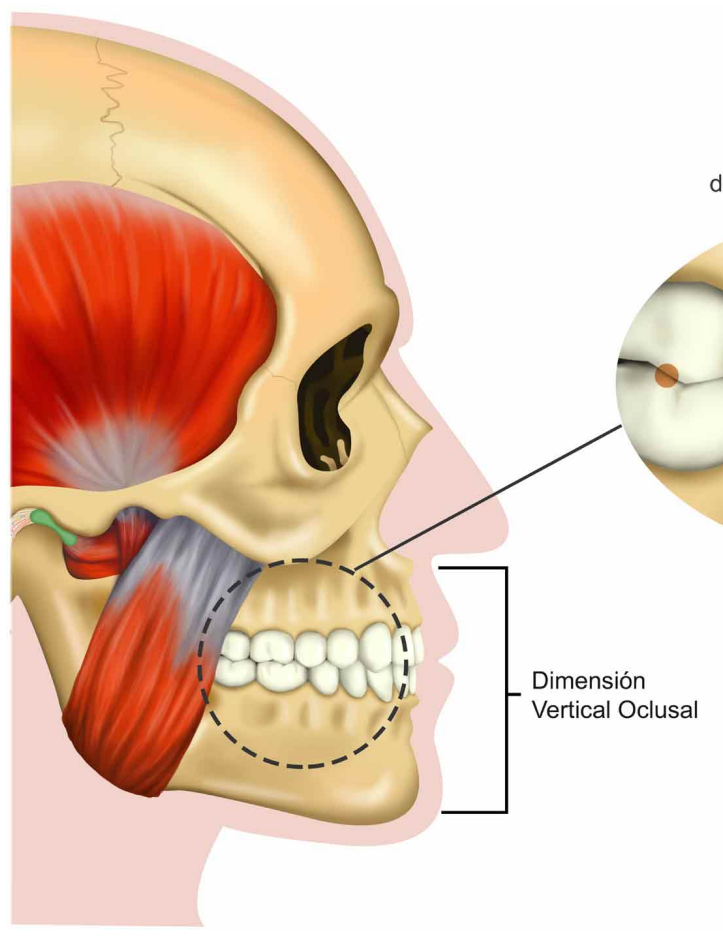

Definido por el determinante anatómico dentario
En la literatura se han descrito diferentes posiciones mandibulares, las cuales están definidas por determinantes anatómicos y fisiológicos, los primeros están representados por la articulación temporomandibular y la oclusión dentaria, y el determinante fisiológico que se refiere a la neuro-musculatura del sistema estomatognático (Manns \& Biotti, 2006), actualmente el estudio de estas posiciones es un tema de gran relevancia, ya que se han utilizado en la evaluación de trastornos temporomandibulares, en investigaciones en el área de la odontología, y en las diferentes etapas de tratamientos de rehabilitación oral y ortodoncia, ya que los tratamientos odontológicos se basan en devolver la función en base a estas posiciones de referencia (Brill \& Tryde; Mohamed \& Christensen; Manns \& Diaz; Walker et al., 2016; Tripodakis et al.; Davies et al.), es por ello que el objetivo de este artículo se basa en describir las posiciones mandibulares más utilizadas como referencia clínica al momento de realizar diferentes procedimientos odontológicos.

\section{Posiciones Mandibulares}

Posición de Máxima Intercuspidación (PMI). Se define como aquella posición mandibular lograda cuando los dientes se encuentran completamente interdigitados, logrando que exista el mayor número de contactos oclusales, estando definida por lo tanto, por el determinante anatómico dentario (Fig. 1) (Manns \& Diaz; Manns \& Biotti; Mohamed \& Christensen; Tripodakis et al.). Algunos autores también la denominan como "oclusión céntrica" (Mohamed \& Christensen; Tripodakis et al.).
Fig. 1. Posición de Máxima Intercuspidación (PMI), definida por el determinante anatómico dentario. Esta posición determina la Dimensión Vertical Oclusal. 
Es importante el análisis de los contactos oclusales que se presentan al momento de realizar esta posición, los cuales son dependientes de la presencia, forma y posición de los dientes (Manns \& Biotti), además, pueden verse alterados por algunos cambios producidos a lo largo de la vida de los individuos, tales como, pérdida de dientes, desgastes oclusales, movimientos dentarios y distintos tipos de tratamientos odontológicos (Miranda et al., 2019), sin embargo, existen contactos de referencia que se han estudiado en sujetos con oclusión normal, demostrándose que la mayoría de estos se ubican a nivel de los primeros molares inferiores y superiores (Watanabe-Kanno \& Abrão, 2012).

Es sabido que la posición de la cabeza altera la vía muscular de cierre para llegar a PMI, modificando el contacto dentario inicial, sin embargo, se ha demostrado que la posición de la cabeza no altera el la relación de contacto en máxima intercuspidación, ya que esta es dependiente de las estructuras dentarias, y no de la musculatura, por lo que los dientes son los encargados de guiar la mandíbula hasta llegar a PMI, permaneciendo esta sin cambios al encontrarse la cabeza y el cuerpo en distintas posiciones (Mc Lean et al., 1973; Mohamed \& Christensen; Makofsky, 1989; Tripodakis et al.).

Esta posición se utiliza como referencia para la realización de cualquier tipo de tratamiento restaurador, considerando su adecuada reproducibilidad y sencilla ejecución (Tripodakis et al.), antes de la planificación de cualquier tratamiento es necesario realizar un correcto diagnóstico oclusal, siendo de gran importancia

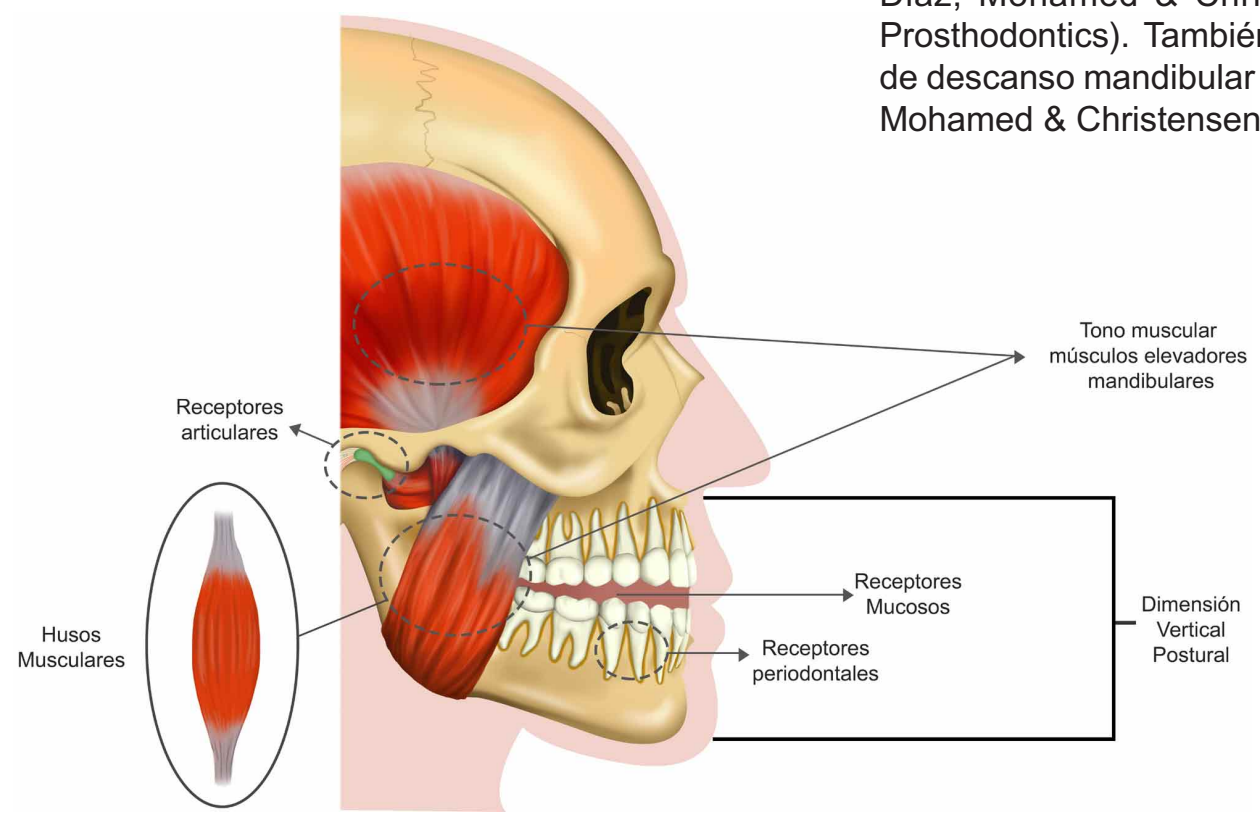

el estudio de la naturaleza de los contactos oclusales producidos en PMI, de tal manera que el objetivo terapéutico se base en devolver la funcionalidad de la manera más compatible posible, asegurando el éxito del tratamiento (Firmani et al., 2013; Anderson \& Myers; Walker et al.). Otro aspecto importante a considerar, es que esta posición ha demostrado no verse alterada con la inserción de planos de mordida, y ser una posición estable y de referencia óptima solo cuando los dientes posteriores están presentes, permitiendo una oclusión estable y reproducible (Tripodakis et al.; Mohamed \& Christensen), por lo que para su registro y reproducción o montaje en articulador en sujetos con oclusión estable, no es necesaria la utilización de algún tipo de dispositivo interoclusal, ya que solo basta con realizar una correcta relación de los modelos de yeso maxilar y mandibular.

La posición de máxima intercuspidación determina la Dimensión Vertical Oclusal (DVO) (Fig. 1), que se define como la altura del tercio inferior de la cara cuando el sujeto se encuentra en esta posición (Manns \& Diaz; Mohamed \& Christensen; The Academy Of Prosthodontics, 2017; Quiroga-del Pozo et al., 2016).

Posición Postural Mandibular (PPM). Se define como aquella relación que adopta la mandíbula con respecto a la maxila cuando el sujeto se encuentra de pie o sentado cómodamente en posición erguida u ortostática, con los labios juntos y los músculos asociados se encuentran en un estado de actividad contractural mínima (Fig. 2), siendo una posición íntegramente dependiente del factor de gravedad y del tono muscular (Manns \& Diaz; Mohamed \& Christensen; The Academy Of Prosthodontics). También se le ha llamado posición de descanso mandibular (Murphy, 1967; Brill \& Tryde; Mohamed \& Christensen; Darling et al., 1984).

Fig. 2. Posición Postural Mandibular (PPM), se observan las principales fuentes periféricas que participan en la mantención del tono muscular, tales como; husos musculares, receptores articulares, mucosos y periodontales. Esta posición determina la Dimensión Vertical Postural. 
Esta posición está definida por el determinante fisiológico neuromuscular, y es independiente del número y posición de los dientes (Manns \& Diaz), siendo el resultado de la interacción de múltiples factores relacionados (Fig. 2), donde participan tanto fuerzas activas como pasivas, las fuerzas activas están representadas por la ligera actividad contráctil presente en los músculos elevadores mandibulares, es decir, el tono muscular, este depende de las señales enviadas por las neuronas del núcleo motor del nervio trigémino en respuesta a los impulsos que reciben de las fuentes periféricas y centrales, las primeras están representadas por los husos musculares y los receptores mucosos, articulares y periodontales, y las fuentes centrales son el sistema límbico y las áreas visuales del cerebro (Murphy; Brill \& Tryde; Mohamed \& Christensen; Manns \& Diaz), por otro lado las fuerzas pasivas están determinadas por la contribución de los músculos elevadores mandibulares, la ATM y el denominado "Espacio de Donders" (Brill \& Tryde; Manns \& Diaz) que se refiere a las áreas de la cavidad oral donde puede surgir presión negativa cuando se está en PPM, donde la lengua se encuentra cumpliendo un rol importante (Brill \& Tryde; Murphy). En cuanto a la participación muscular es importante reconocer que sus propiedades viscoelásticas son las que se encargan de resistir el movimiento, permitiendo esta tensión pasiva que se mantiene en PPM (Brill \& Tryde; Mohamed \& Christensen), la ATM también presenta una participación pasiva, ya que la posición del cóndilo depende de las fuerzas externas que trabajan en la articulación y las propiedades viscoelásticas de las estructuras periarticulares e intraarticulares (Oishi, 1967).

Se considera la posición menos estable, ya que puede ir cambiando durante las distintas etapas de vida de los individuos, esto debido a que puede verse influenciada por un importante grupo de factores, dentro de los cuales se encuentran; la postura del cuerpo, dolores musculares o relacionados a la ATM, maloclusiones, desarrollo facial, enfermedades que afecten el sistema locomotor mandibular, e incluso se ha estudiado que el miedo, estrés y ansiedad pueden afectar esta posición al alterar la actividad muscular (Perry et al., 1960; Mohamed \& Christensen; Huamani-Cantoral et al., 2018; Murphy).

La PPM determina la Dimensión Vertical Postural (DVP) (Fig. 2), que es la altura del tercio inferior de la cara cuando el sujeto se encuentra en esta posición (Manns \& Diaz; Mohamed \& Christensen).
Al realizar la PPM, existe un espacio entre las superficies oclusales de los dientes maxilares y mandibulares, el cual ha sido denominado como "espacio libre o de inoclusión fisiológico", el cual normalmente mide de 1 a $3 \mathrm{~mm}$ (Mohamed \& Christensen; Manns \& Diaz; The Academy Of Prosthodontics), y se dice que tiene forma de cuña, ya que los incisivos y molares antagonistas se encuentran separados en una proporción de 3:2 respectivamente (Tingey et al., 2001), además estudios han demostrado que esta distancia varía de acuerdo a la posición de la cabeza (Cohen, 1957; Darling et al.), este espacio es utilizado para la determinación de la Dimensión Vertical, a través de la PPM como referencia (Sudhir et al., 2015).

Cuando un sujeto pierde dientes o sufre desgastes oclusales, la DVO se altera, jugando aquí el diagnóstico un rol importante, ya que su evaluación se considera como el punto de inicio en todo tratamiento rehabilitador, cuyo objetivo se basa en reestablecer la DVO (Huamani-Cantoral et al.). Determinar la Dimensión Vertical, en sujetos desdentados se considera una tarea difícil, y una etapa esencial de todo tratamiento protésico, para ello hasta el día de hoy se han desarrollado distintas técnicas, de las cuales la gran mayoría se basa en un equilibrio adecuado entre la DVO y DVP, utilizando como referencia el espacio libre de inoclusión, estas técnicas deben presentar un protocolo adecuado donde es fundamental que el paciente se encuentre en una postura vertical adecuada y sin ningún tipo de sintomatología, ya que estas son situaciones que modifican el registro, (Ramirez et al., 2013; Toolson \& Smith, 1982; Huamani-Cantoral et al.). Al haber sido demostrado que la postura del cuerpo y cabeza es un factor que altera la PPM, es importante que se considere este aspecto al momento de realizar su evaluación (Darling et al.; Mc Lean et al.; Tingey et al.), las técnicas para su registro incluyen el uso de la PPM, fonética, deglución, cefalometría, dimensiones faciales, entre otras, sin embargo, ninguna por si sola es capaz de determinar una DVO precisa en un paciente desdentado, por lo que para la determinación de una DVO más exacta se recomienda que se utilice una combinación de técnicas, de tal manera que puedan complementarse entre sí (Toolson \& Smith; Sudhir et al.; Igic et al., 2015; Geerts et al., 2004; Nagpal et al., 2013).

Relación Céntrica Fisiológica (RCF). Se ha descrito como una de las posiciones que presenta más controversia en la literatura, tanto en su definición 
como en el procedimiento para su registro (Orozco et al., 2008). Es una posición que está determinada por las relaciones espaciales entre los componentes de la ATM (Mohamed \& Christensen), y ha sido definida como la posición en la cual el cóndilo de la mandíbula está localizado en su posición fisiológicamente más superior, anterior y media dentro de la fosa mandibular, y la porción media y más delgada del disco articular se encuentra interpuesta entre la vertiente anterior del cóndilo y la vertiente posterior de la eminencia articular del hueso temporal (Fig. 3) (Biotti et al., 2006; Manns \& Biotti; Wiens et al., 2018). Corresponde a aquella posición más estable de la mandíbula, en la cual las superficies y tejidos articulares están alineados de tal forma que la fuerza muscular elevadora mandibular potencialmente no produce daño articular (Biotti et al.), es una posición repetible e independiente del contacto dentario y está restringida a un movimiento rotacional puro de la cabeza condilar en torno a un eje de bisagra, permitiendo que la mandíbula durante esa fase de movimiento describa un arco (Wiens et al.; Davies \& Gray).

Se considera una posición de referencia reproducible, de gran interés para la planificación de los tratamientos clínicos (The Academy Of Prosthodontics) y útil para el montaje de modelos en

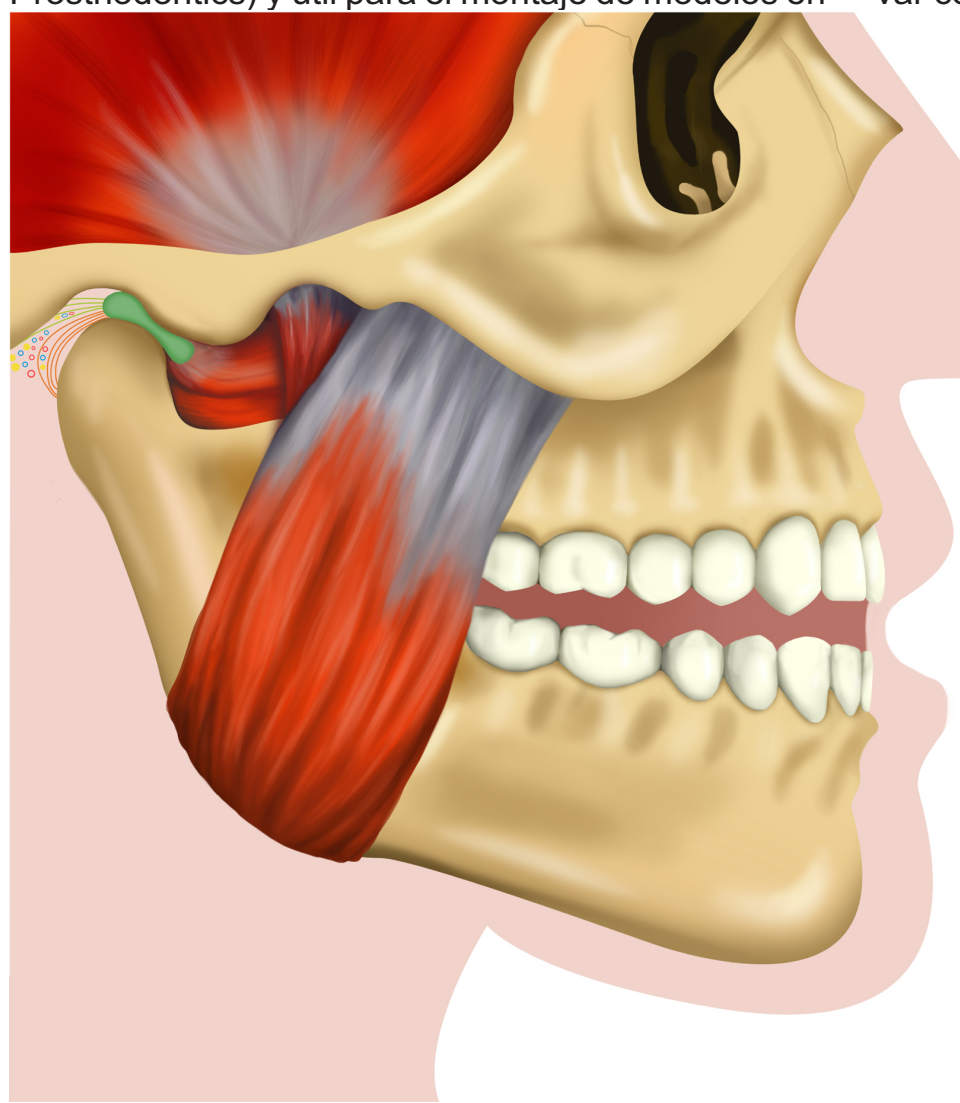

articulador (Wiens et al.), que va adquiriendo mayor importancia a medida que aumenta el número de unidades oclusales a rehabilitar en el tratamiento (Goldstein et al., 2017). Al ser la posición ideal de los cóndilos en la fosa mandibular, se requiere que todos los tratamientos rehabilitadores en pacientes en los que se reestablecerá la oclusión por completo, se enfoquen en la RCF como punto de referencia, ya que se debe ajustar la oclusión poco a poco hacia esta relación, hasta que se logre la adaptación fisiológica, otorgando al paciente una oclusión funcional y estable (Pokorny et al., 2008).

Debido a su relevancia en la práctica clínica, a lo largo de los años se han ido desarrollado diferentes técnicas para su registro, las cuales han sido analizadas y comparadas en diferentes estudios, muchos de los cuales concluyen que la técnica de manipulación mandibular de Dawson es actualmente una de las más reproducibles, y se considera un método consistente para el registro de RCF (Orozco et al.), esta técnica proporciona la relajación de los músculos masticatorios mediante la manipulación manual no forzada de la mandíbula. Se requiere de una deprogramación previa, de tal manera de borrar el engrama neuromuscular existente y lograr llevar correctamente a la ATM a relación céntrica fisiológica, logrando obtener un resultado más preciso (Stafeev et al., 2018). Esto se puede realizar a través de: Jit de Lucía (Lucia, 1964), el uso de férulas (Forsell et al., 1999), u otras técnicas, para lograr interrumpir la propiocepción y lograr un registro más exacto (Wilson \& Banerjee, 2004).
Fig. 3. Relación Céntrica Fisiológica (RCF), se observa el cóndilo en su posición más superior, anterior y media en la fosa mandibular, con la porción media y más delgada del disco articular interpuesta entre la vertiente anterior del cóndilo y la vertiente posterior de la eminencia articular del hueso temporal. 
Posición Retruida Ligamentosa (PRL). También se le ha llamado Relación Céntrica Ligamentosa (Biotti et al.). Se define como aquella relación entre la mandíbula y la maxila cuando los cóndilos se encuentran en su posición más retruida en la fosa mandibular, determinada por la tensión de la banda horizontal medial del ligamento temporomandibular (Fig. 4). Los movimientos de apertura y cierre realizados con los cóndilos en su posición más retruida son movimientos de bisagra puros. La PRL no es una posición funcional, debido a debido a que es una posición extrema que raramente se observa durante la masticación y deglución (Brill \& Tryde; Manns \& Diaz).

Se ha observado que esta posición no presenta variaciones en relación a la postura corporal (Helkimo et al., 1971). Estudios pasados indican que al ser una posición que depende del determinante ligamentoso, es estable y reproducible, por lo que se ha considerado como una posición de referencia adecuada para el análisis funcional de la oclusión, y una referencia clínica importante para el montaje de modelos en el articulador (Posselt, 1952; Helkimo et al.; Manns \& Diaz; Brill \& Tryde), permitiendo la realización del movimiento mandibular para evaluar contactos en la realización de restauraciones (Wilson \& Banerjee). Sin embargo, actualmente ha disminuido su uso como posición mandibular de referencia debido a su condición límite y no funcional (Biotti et al.).
Para su registro se requiere la manipulación de la mandíbula por parte del operador, de tal manera que logre el movimiento de las cabezas condilares por la pendiente posterior de la fosa mandibular y giren alrededor de los ligamentos temporomandibulares, siendo limitados por estos, se debe tener especial cuidado de no generar dolor o molestias en el paciente (Biotti et al.; Helkimo et al.; Wilson \& Banerjee). Existen muchas técnicas para lograr el registro de esta posición, las cuales pueden requerir el uso de cera para registros, pastas de óxido de zinc, resinas acrílicas, etc. (Wilson \& Banerjee) en pacientes que tienen dificultades para lograr la posición, se requiere la deprogramación neuromuscular de los músculos masticatorios.

Posición Retruida de Contacto (PRC). Esta es una posición de difícil evaluación clínica directa, por lo que generalmente se examina en modelos articulados ya sea en PRL o RCF. La PRC se describe como la posición mandibular cuando se producen los primeros contactos dentarios y la ATM está en una posición retruida.

En la literatura se definen dos tipos de posiciones retruidas de contacto:

Posición retruida de contacto forzada: Se refiere a la relación de contacto oclusal que adopta la mandíbula cuando cierra en posición retruida o posterior for-

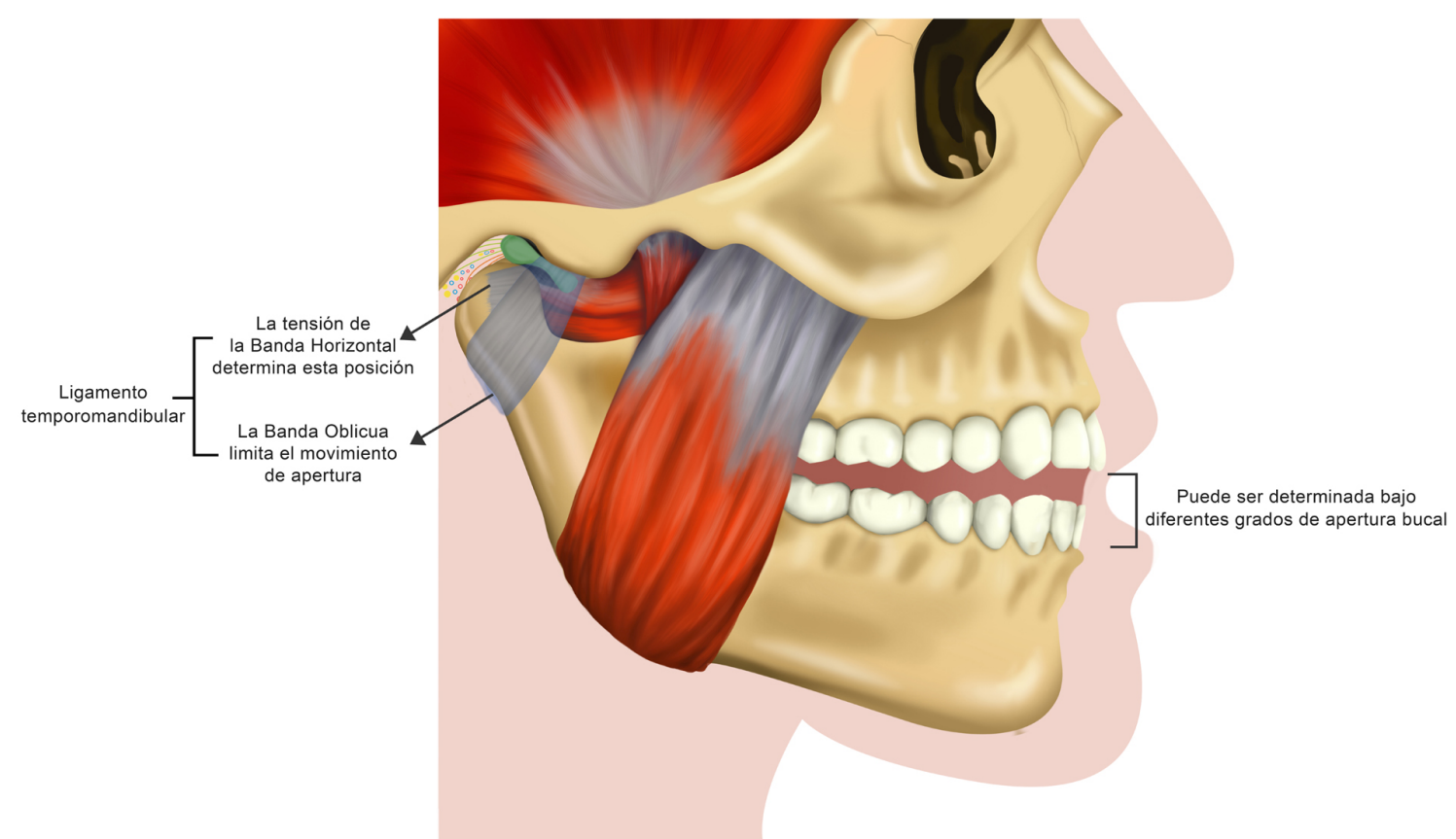

Fig. 4. Posición Retruida Ligamentosa (PRL), se observa el cóndilo de la mandíbula en su posición más retruida en la fosa mandibular, y la tensión de la banda horizontal medial del ligamento temporomandibular, que limita esta posición. 
zada, es decir, con sus cóndilos en posición retruida ligamentosa, hasta encontrar el contacto inicial de uno o más dientes, a lo largo del camino de cierre retruido ligamentoso (Fig. 5). También se ha denominado como posición de contacto en relación céntrica ligamentosa (Manns \& Biotti).

Posselt encontró que sólo en aproximadamente el $10 \%$ de los individuos dentados, esta posición coincidiría con PMI, y en la otra parte de la población de estudio se encontró esta posición entre 0,5 - $2 \mathrm{~mm}$ hacia posterior de PMI, otro estudio demostró que esta coincidencia fue observada en un $29 \%$ de la población estudiada, encontrándose en su mayoría una PRC aproximadamente $1 \mathrm{~mm}$ posterior a PMI (Pullinger et al., 1988). Estos hallazgos demuestran el bajo porcentaje de coincidencia de estas posiciones y sugieren una PRC menor o igual a $2 \mathrm{~mm}$ posterior a PMI podría tener un efecto de protección biomecánica para las ATM (Manns \& Biotti). El porcentaje de coincidencia que existe en estas dos posiciones aún es discutible, ya que, al ser PRC una posición de contacto dentario definida por un ligamento, que se obtiene cuando las ATM están en PRL, luego de la manipulación forzada de la mandíbula, no se considera fisiológica su coincidencia con PMI, por lo que se hacen necesarias nuevas investigaciones, con metodologías que permitan resultados más precisos, de tal manera de determinar si ese porcentaje de coincidencia realmente existe.
Posición retruida de contacto no forzada: Relación de contacto oclusal que alcanza la mandíbula cuando cierra en posición retruida no forzada, es decir; con sus cóndilos en relación céntrica fisiológica (RCF) hasta lograr los contactos primeros contactos oclusales. También se conoce como posición de contacto en relación céntrica fisiológica (Manns \& Biotti), o como contacto prematuro en relación céntrica, esta posición determina el punto final del arco de cierre mandibular en el eje de bisagra terminal o RCF, el cual puede ser un simple contacto, o bien puede coincidir con PMI (Davies \& Gray). La distancia entre la posición retruida de contacto no forzada y PMI ha sido denominada como deslizamiento en céntrica (Keshvad \& Winstanley, 2001).

Posición Muscular de Contacto (PMC): También se conoce como posición miocéntrica (Biotti et al.). Se refiere a la posición que alcanza la mandíbula cuando se eleva por una contracción isotónica de los músculos elevadores mandibulares a lo largo del camino muscular de cierre, hasta encontrar el primer contacto oclusal (Fig. 6) (Mohamed \& Christensen; Biotti et al.), desde el punto de vista fisiológico esta posición debería ser coincidente con PMI cuando el sujeto se encuentre en posición erguida, determinando esto una armonía funcional perfecta en el sistema estomatognático, donde no existan interferencias dentarias en el movimiento de cierre mandibular, tenien-

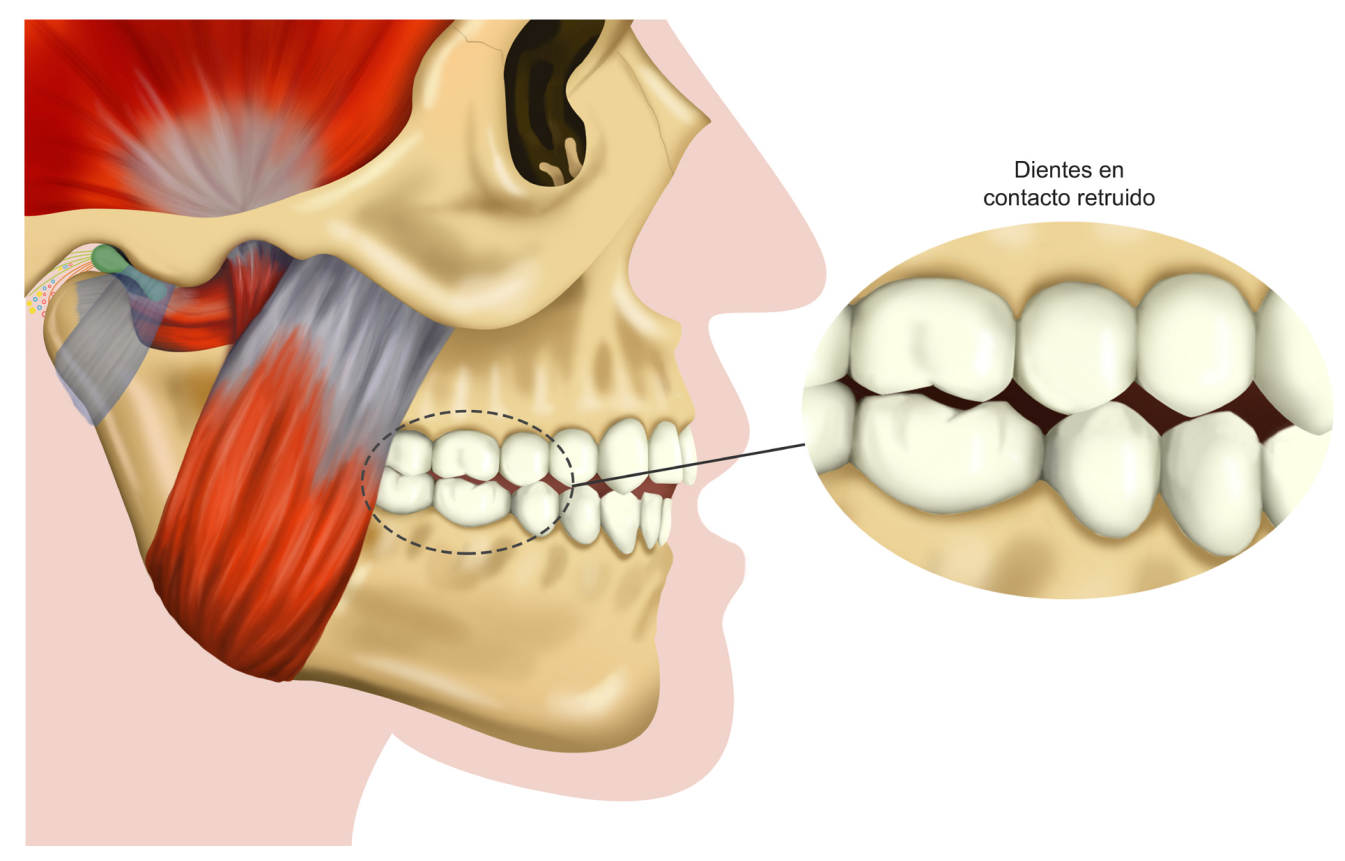

Fig. 5. Posición Retruida de Contacto (PRC) Forzada, se observa el cóndilo de la mandíbula en su posición más posterior en la fosa mandibular y los dientes en contacto. 
do en cuenta que PMI enfatiza una relación anatómica dentaria, mientras que PMC designa el aspecto fisiológico muscular de la misma posición mandibular (Biotti et al.; Manns \& Diaz; Mohamed \& Christensen).

En PMC el cóndilo de la mandíbula se encuentra en una posición intermedia en la fosa mandibular, lo cual se logra mediante la participación de fuerzas activas y pasivas que actúan sobre las ATM (Fig. 6) (Brill \& Tryde). Esta posición es regulada a través las señales enviadas por los receptores periodontales hacia el núcleo motor del trigémino al momento del contacto dentario (Fig. 6), lo cual permite que la actividad muscular se ajuste a estos impulsos manteniendo PMC, sin embargo, este mecanismo de control se pierde con la ausencia de dientes, por lo que no es una posición estable (Manns \& Diaz; Brill \& Tryde), además se reconoce que la postura corporal del sujeto es un factores que puede modificar la PMC (Makofsky; Tripodakis et al.).

En determinadas situaciones clínicas, los contactos prematuros, determinan la generación de nuevos engramas neuromusculares que pueden desviar la mandíbula durante el camino muscular de cierre hacia una PMI de acomodo que no sea coincidente con

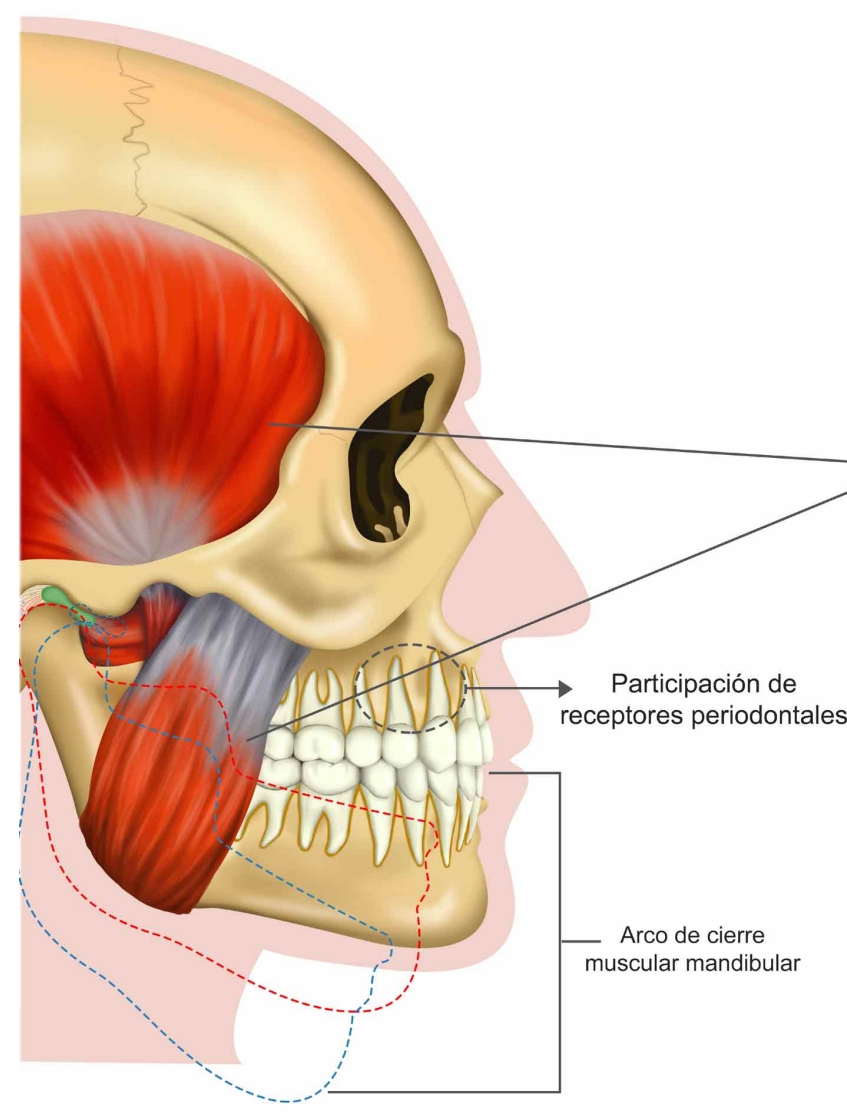

PMC, en estas situaciones se hace necesaria la deprogramación neuromuscular para suprimir los engramas (Biotti et al.).

En la rehabilitación de un paciente, cuando se requiere generar una oclusión nueva, idealmente PMC debería coincidir con PMI y RCF (Manns \& Diaz). Para el registro de esta posición, en ocasiones es necesario utilizar cera de registro o rodetes de altura, y no es necesaria la manipulación por parte del operador, ya que solo basta con indicarle al paciente que cierre a través de su arco de cierre habitual hasta llegar al contacto dentario (Tripodakis et al.; Makofsky et al., 1991), cuando esta posición es coincidente con RCF, su registro debe realizarse a través de las técnicas ya descritas para esa posición.

\section{CONCLUSIÓN}

El estudio de las posiciones mandibulares proporciona una base importante en el área de la fisiología oral, su conocimiento y comprensión es esencial para el diagnóstico, planificación y pronóstico de diferentes tratamientos odontológicos, su interés radica en la base fisiológica que proporcionan para su utilización adecuada bajo distintas circunstancias clínicas, para ello es importante el análisis de las características más importantes que definen su uso, tales como su estabilidad y reproducibilidad, que son fundamentales a considerar al momento de la toma de decisiones clínicas.
Fig. 6. Posición Muscular de Contacto (PMC), esta posición está determinada por el contacto dentario y la participación activa de los músculos elevadores mandibulares. Se observa el arco de cierre muscular mandibular necesario para llegar a esta posición, representado por la mandíbula en 20 $\mathrm{mm}$ aprox. de apertura en rojo, y en azul una apertura máxima, donde se observa la traslación del cóndilo. 
VENEGAS, C.; FARFÁN, C. \& FUENTES, R. Mandibular Positions of Clinical Reference. Narrative description. Int. J. Odontostomat.,15(2):387-396, 2021.

ABSTRACT: Mandibular positions are defined as the existing relation between mandibula and maxilla during its function, or it can also be defined as clinical reference positions that may be comparable with other positions or movements. A narrative description of the mandibular reference positions of greatest clinical interest was made, where some of their main characteristics were defined; (1) maximum intercuspal position, is defined by de dental anatomical determinant, it has an adequate reproduction and simple execution, (2) the mandibular postural position, it is the least stable, and it is defined by the neuromuscular physiological elements, being the result of the interaction of multiple factors related, (3) the physiological centric relation is considered the most stable position of the mandible, being easy to repeat and independent of dental contact, (4) the retruded ligamentous position, is an extreme position so it is not considered physiological, and it is determinated by the tension of the medial horizontal band of the temporomandibular ligament, (5) retruded contact position is described as the mandibular position when the first dental contacts are produced and the temporomandibular joint (TMJ) is in a retruded position, it is divided into forced and nonforced, (6) the muscular contact position is a position regulated through different mechanisms that allows its stability. These positions provide a clinical reference by means of which different parameters required while diagnosing and planning treatments can be evaluated, this is why it is important for the Specialist to have specific knowledge about the characteristics that correspond to each one of them, such as its stability and reproducibility, which are fundamental at the time of its execution.

KEY WORDS: Maximum intercuspal position, mandibular postural position, muscular contact position, retruded contact position, retruded ligamentous position, physiological centric relation, vertical dimension.

\section{REFERENCIAS BIBLIOGRÁFICAS}

Apodaca, A. Fundamentos de Oclusión. Ciudad de México, Instituto Politécnico Nacional, 2004.

Biotti, J.; Manns, A.; González, C. \& Loeff, N. Glosario de Oclusión dentaria y trastornos temporomandibulares. Colombia, AMOLCA, 2006.

Brill, N. \& Tryde, G. Physiology of Mandibular Positions. Front. Oral Physiol., 199-237, 1974.

Cohen, S. A cephalometric study of rest position in edentulous persons: Influence of variations in head position. J. Prosthet. Dent., 7(4):467-72, 1957.

Darling, D. W.; Kraus, S. \& Glasheen-Wray, M. B. Relationship of head posture and the rest position of the mandible. J. Prosthet. Dent., 52:111-5, 1984.
Davies, S. J. \& Gray, R. M. J. Occlusion: The examination and recording of the occlusion: why and how. Br. Dent. J., 191(6):291302, 2001.

Davies, S. J.; Gray, R. M.; Sandler, P. J. \& O'Brien, K. D. Orthodontics and occlusion. Br. Dent. J., 191(10):539-49, 2001.

Firmani, M.; Becerra, N.; Sotomayor, C.; Flores, G. \& Salinas, J. C. Oclusión terapéutica: Desde las escuelas de oclusión a la Odontología Basada en Evidencia. Rev. Clin. Periodoncia Implantol. Rehabil. Oral, 6(2): 90-5, 2013.

Forsell, H.; Kalso, E.; Koskela, P.; Vehmanen, R.; Puukka, P. \& Alanen, P. Occlusal treatments in temporomandibular disorders: a qualitative systematic review of randomised controlled trials. Pain, 83:549-61, 1999.

Geerts, G. A.; Stuhlinger, M. E. \& Nel, D. G. A comparison of the accuracy of two methods used by pre-doctoral students to measure vertical dimensión. J. Prosthet. Dent., 91(1):59-66, 2004.

Goldstein, G.; Andrawis, M.; Choi, M.; Wiens, J. \& Janal, M. N. A survey to determine agreement regarding the definition of centric relation. J. Prosthet. Dent., 117(3):426-9, 2017.

Helkimo, M.; Ingervall, B. \& Carlsson, G. E. Variation of Retruded and Muscular Position of Mandible Under Different Recording Conditions. Acta Odontol. Scand., 29(4):423-37, 1971.

Huamani-Cantoral, J.; Huamani-Echaccaya, J. \& Alvarado-Menacho, S. Rehabilitación oral en paciente con alteración de la dimensión vertical oclusal aplicando un enfoque multidisciplinario. Rev. Estomatol. Herediana, 28(1):44-55, 2018.

Igic', M.; Krunic', N.; Aleksov, L.; Kostic', M.; Igic'. A.; Petrovic', M. B. Dacic', S.; Igic', S. \& Igic', A. Determination of vertical dimension of occlusion by using the phonetic vowel "O" and "E". Vojnosanit Pregl., 72(2):123-31, 2015.

Keshvad, A. \& Winstanley, R. B. An appraisal of the literature on centric relation. Part III. J. Oral Rehabil., 28:55-63, 2001.

Lucia, V. O. Technique for recording centric relation. J. Prosthet. Dent., 14(3):492-505, 1964

Makofsky, H. W. The effect of head posture on muscle contact position: the sliding cranium theory. CRANIO, 7(4):286-92, 1989.

Makofsky, H. W.; Sexton, T. R.; Diamond, D. Z. \& Sexton, M. T. The effect of head posture on muscle contact position using the t-scan system of occlusal analysis. CRANIO, 9(4):316-21, 1991.

Manns, A. \& Biotti, J. Manual Práctico de Oclusión Dentaria. 2a ed. Caracas Venezuela, AMOLCA, 2006.

Manns, A. \& Diaz, G. Sistema Estomatognático. Santiago de Chile, Gráfica Almagro, Universidad de Chile, 1988.

Mc Lean, L. F.; Brenman, H. S. \& Friedman, M. G. F. Effects of changing body position on dental occlusion. J. Dent. Res., 52(5):1041-5, 1973.

Miranda, F.; Massaro, C.; Janson, G.; de Freitas, M. R.; Henriques, J. F. C.; Lauris, J. R. P. \& Garib, D. Aging of the normal occlusion. Eur. J. Orthod., 41(2):196-203, 2019.

Mohamed, S. E. \& Christensen, L. V. Mandibular reference positions. J. Oral Rehabil., 12(4):355-67, 1985.

Moorrees, C. Occlusion. J. Periodontol., 38(6):751-60, 1967.

Murphy, W. M. Rest position of the mandible. J. Prosthet. Dent., 17(4):329-32, 1967.

Nagpal, A.; Parkash, H.; Bhargava, A. \& Chittaranjan, B. Reliability of different facial measurements for determination of vertical dimension of occlusion in edentulous using accepted facial dimensions recorded from dentulous subjects. J. Indian Prosthodont. Soc., 14(3):233-42, 2013.

Oishi, T. A Study on the anatomical structure of temporomandibular joint from the standpoint of mandibular movement. Nihon Hotetsu Shika Gakkai Zasshi, 11(2):197-220, 1967.

Orozco, A.; Arroyo, G.; Martínez de Fuentes, R.; Ventura de la Torre, J.; Cañadas, D. \& Jiménez, E. Relación céntrica: revisión de conceptos y técnicas para su registro. Parte II. Av. Odontoestomatol., 24(6):369-76, 2008. 
Perry, H. T.; Lammie, G. A.; Main, J. \& Teuscher, G. W. Occlusion in a stress situation. J. Am. Dent. Assoc., 60(5):626-33, 1960.

Pokorny, P. H.; Wiens, J. P. \& Litvak, H. Occlusion for fixed prosthodontics: A historical perspective of the gnathological influence. J. Prosthet. Dent., 99(4):299-313, 2008.

Posselt, U. Studies in the mobility of the human mandible. Acta Odontol. Scand., 10:13-150, 1952.

Pullinger, A. G.; Seligman, D. A. \& Solberg, W. K. Temporomandibular disorders. Part I: Functional status, dentomorphologic features, and sex differences in a nonpatient population. J. Prosthet. Dent., 59(2):228-35, 1988.

Quiroga-del Pozo, R.; Sierra-Fuentes, M.; del Pozo-Bassi, J. \& Quiroga-Aravena, R. Dimensión vertical oclusal: comparación de 2 métodos cefalométricos. Rev. Clin. Periodoncia Implantol. Rehabil. Oral, 9(3):264-70, 2016.

Ramirez, L. M.; Echeverría, P.; Zea, F. J. \& Ballesteros, L. E. Vertical dimension on edentulous patient: relationship with symptoms reported. Int. J. Morphol., 31(2):672-80, 2013.

Stafeev, A. A.; Solovyev, S. I.; Petrov, P. O. \& Khizhuk, A. V. The precision of central jaw relationship estimation in restorative dentistry. Stomatologiia (Mosk.), 97(4):31-6, 2018.

Sudhir, N.; Chittaranjan, B.; Kumar, B. A.; Taruna, M. \& Kumar, M. P. Digital cephalometric tracings by pro-ceph $v 3$ software for comparative analyses of vertical dimension in edentulous patients. J. Clin. Diagn. Res., 9(5):ZC01-5, 2015.

The Academy Of Prosthodontics. The Glossary of Prosthodontic Terms. Ninth Edition. J. Prosthet. Dent., 117(5):e1-e105, 2017.

Tingey, E. M.; Buschang, P. H. \& Throckmorton, G. S. Mandibular rest position: A reliable position influenced by head support and body posture. Am. J. Orthod. Dentofacial Orthop., 120(6):61422, 2001

Toolson, L. B. \& Smith, D. E. Clinical measurement and evaluation of vertical dimension. J. Prosthet. Dent., 47(3):236-41, 1982.

Tripodakis, A. P.; Smulow, J. B.; Mehta, N. R. \& Clark, R. E. Clinical study of location and reproducibility of three mandibular positions in relation to body posture and muscle function. J. Prosthet. Dent., 73(2):190-8, 1995.

Walker, T. F.; Broadwell, B. K. \& Noujeim, M. E. MRI assessment of temporomandibular disc position among various mandibular positions: a pilot study. CRANIO, 35(1):10-4, 2016.

Watanabe-Kanno, G. A. \& Abrão, J. Study of the number of occlusal contacts in maximum intercuspation before orthodontic treatment in subjects with Angle Class I and Class II Division 1 malocclusion. Dental Press J. Orthod., 17(1):138-47, 2012.

Wiens, J. P.; Goldstein, G. R.; Andrawis, M.; Choi, M. \& Priebe, J. W. Defining centric relation. J. Prosthet. Dent., 120(1):114-22, 2018.

Wilson, P. H. R. \& Banerjee, A. Verifiable CPD paper: Recording the retruded contact position: a review of clinical techniques. Br. Dent. J., 196(7):395-402, 2004.
Dirección para correspondencia:

Prof. Dr. Ramón Fuentes Fernández

Centro de Investigación en Ciencias Odontológicas

Facultad de Odontología

Universidad de La Frontera

Av. Francisco Salazar 1145

Temuco

CHILE

E-mail: ramon.fuentes@ufrontera.cl 